\title{
Apparent misalignment of oblique coplanar bars in depth
}

\author{
R. H. DAY \\ La Trabe University, Bundoora, Victoria, Australia \\ and \\ ANDREA L. PARKER HALFORD \\ Deakin University, Burwood, Victoria, Australia
}

\begin{abstract}
Following preliminary observations of apparent misalignment of coplanar surfaces in a threedimensional (3-D) form of the Poggendorff figure viewed in depth, the effect was investigated in five experiments and compared with the 2-D Poggendorff effect in the same object in a sixth. The effect in depth occurred with the complete object when it was viewed binocularly but did not do so with the oblique bars alone or when the object was viewed monocularly. The effect did not vary with oblique-parallel angles of $30^{\circ}, 45^{\circ}$, and $60^{\circ}$ and was absent when the angle was $90^{\circ}$. It varied as a function of the distance between the parallels, but was unaffected by a regular pattern on the oblique bars. A smaller 2-D Poggendorff effect occurred when the upper edges of the object were viewed from above. Although the depth effect was robust, its variance was high compared with that of the 2-D effect, indicating that acuity for misalignments of oblique elements in depth is poor. An explanation of apparent misalignment of oblique elements in 2-D and 3-D space in terms of a perceptual compromise between alignment in an oblique axis and separation relative to the axes of the parallels is proposed.
\end{abstract}

The results from two recent series of experiments have shown that illusions of extent and misalignment occur in egocentric depth with three-dimensional (3-D) stimulus objects. Gordon, Day, and Dorward (1993) showed that when two identical objects were positioned with their centers $1.6 \mathrm{~m}$ from the observer so that the surface of one was concave and that of the other convex, the center of the concave surface appeared nearer than that of the convex surface. The difference in apparent distance was about $2 \%$ of the viewing distance for right-angle objects and about 5\% for hemicylindrical objects. Daniels and Gordon (1993) have now reported a misalignment effect for coplanar bars positioned obliquely in depth. When the gap between the bars was occluded by a frontoparallel vertical rectangle nearer to the observer, the bars appeared to be misaligned, with the farther bar apparently too far back for alignment with the nearer.

The first of these two illusions in the third dimension bears some resemblance to the Müller-Lyer illusion of extent that occurs in spaces terminating in inward- and outward-directed angles and other elements. In the depth illusion, the spaces between observer and objects termi-

The research reported in this paper was supported by an Australian Research Council grant to the first author. The assistance of Richard Hobbs and Alan Clews in the construction of apparatus, and of Rosemary Williams and Vladimir Kohout in illustrations and photography is gratefully acknowledged. Address all correspondence to R. H. Day, Department of Psychology, La Trobe University, Bundoora, 3083, Victoria, Australia.

-Accepted by previous editor, Charles W. Eriksen nated in outward- and inward-directed 3-D objects. It can be noted that a 2-D form of this egocentric-extent illusion has also been described (Day \& Parks, 1989); two lines of equal length drawn on the floor and extending outward from the observer and terminating in inward and outward angles appeared to be different in length. The illusion described by Daniels and Gordon (1993) is not unlike the 2-D Poggendorff illusion in that the bars appear to be displaced from their common oblique axis with one appearing too far in depth for alignment and the other too far.

Following two recent series of experiments involving a new version of the 2-D Poggendorff figure consisting simply of two oblique bars (Day \& Parker, in press; Day, Stecher, \& Parker, 1993), it was concluded that the Poggendorff effect is essentially an illusion of position. Although the oblique transversals of the conventional figure are positioned in the same axis, they are positioned high and low relative to both the visual field and the parallel lines that separate them. Therefore, it was proposed that the collinear positions of the transversals are compromised in perception by their vertical separation relative to field and parallels. The outcome is the slight but persistent apparent misalignment of the oblique transversals.

Given these terms, it is reasonable to expect that a similar perceptual compromise would occur with two coplanar oblique bars in depth intersecting a pair of parallel sagittal bars (see Figure 1). Although the coplanar bars in Figure 1 are positioned in the same oblique axis, they are positioned relatively near and far in the visual field and relative to the sagittal parallels. Preliminary infor- 
mal observations indicated that a misalignment effect in depth does in fact occur. The primary purpose of the experiments reported here was to confirm and measure this effect and to explore some of its determinants.

Six experiments are described. Experiment 1 was concerned with confirming the preliminary observation of a Poggendorff effect in depth with a 3-D model of the conventional figure viewed in depth, Experiments 2-5 with whether some of the main determinants of the 2-D illusion also influence the 3-D effect, and Experiment 6 with a comparison of the 2-D and 3-D effects in the same object.

\section{GENERAL METHOD}

Since the source of subjects, the apparatus, and the procedure were the same for the six experiments, they can conveniently be described first.

\section{Subjects}

Separate groups of 12 subjects consisting mainly of undergraduates were recruited for each of the six experiments by placing announcements on bulletin boards and through personal contact. Undergraduate subjects were paid for their participation. Both sexes were represented in all the experiments, although the numbers of men and women were not necessarily equal. Inspection of the data has indicated that there were no consistent differences between the sexes.

\section{Apparatus and Viewing Conditions}

The apparatus and viewing conditions are shown in Figure 1. The arrangements were deliberately simple and designed to allow quick, easy manipulation and maximum flexibility in the number of components and their linear and angular dimensions. The stimulus objects shown in Figure 1A were constructed from aluminum bars $25 \mathrm{~mm}$ wide and $1.5 \mathrm{~mm}$ thick and painted matte white. The parallel bars were $250 \mathrm{~mm}$ long and the oblique transversal bars $140 \mathrm{~mm}$ long. The bars could be positioned and held firmly in place by means of 5-mm steel needles projecting from their lower edges. The needles were fitted tightly into holes drilled into the $1.5-\mathrm{mm}$ edges, $20 \mathrm{~mm}$ from each end. When the needles were pressed into the cork surface of the platform, the bars were held firmly in place at right angles to the surface. The platform (Figure 1) was $75.5 \mathrm{~cm}$ wide and $72 \mathrm{~cm}$ deep, and it was backed by a vertical section also $74.5 \mathrm{~cm}$ wide and $30 \mathrm{~cm}$ high. It was surfaced in finely textured, medium brown cork $5 \mathrm{~mm}$ thick. The vertical backing was of compressed fiber board with a finely textured medium brown surface, its color approximating Munsell 10 YR 7/4. The platform was mounted on legs and placed on a table. Illumination was provided by an overhead reading lamp positioned in order to minimize shadows. The subjects viewed the stimulus objects with their heads positioned on a chinrest. The latter was adjusted in height so that only the object itself, never the surface on which it rested, was visible. Thus the white stimulus object was clearly visible in depth against the medium brown vertical backing of the platform. The viewing distance to the near ends of the parallels was always $100 \mathrm{~cm}$, with the ends $13 \mathrm{~cm}$ from the near edge of the platform as shown in Figure 1A. It is emphasized that since the surface of the platform was not visible the guide lines on the surface for angles and separation of the bars could not be seen.

\section{Procedure}

The subjects were shown the experimental arrangement and clearly instructed in the requirements of the task. The transversal bar on the right was held by the experimenter by its outer end at the same angle in depth as that of the bar on the left. The experimenter stood
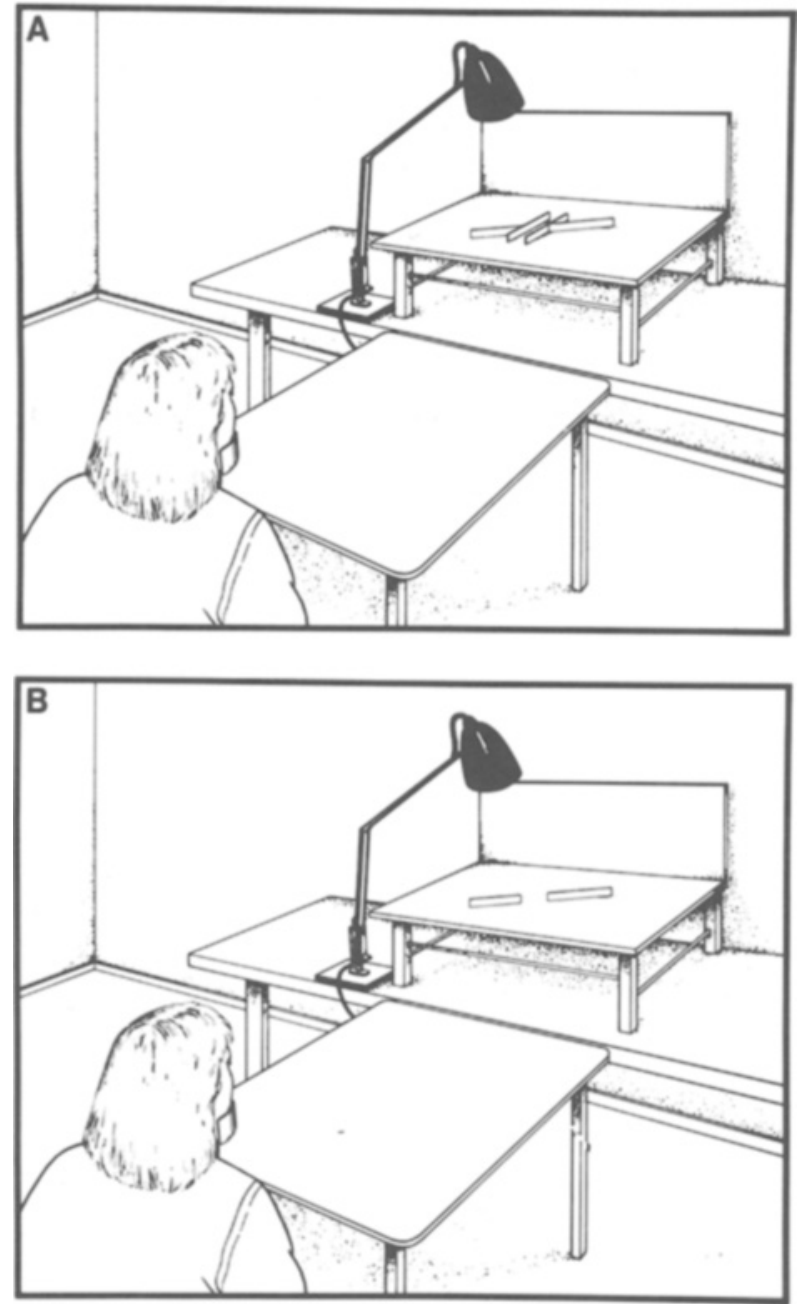

Figure 1. Stimulus objects with (A) and without (B) the parallel bars and the viewing conditions for Experiments 1, 2, 3, 4, and 5 . For Experiment 6, the subjects stood on a low platform and looked down on the object from directly above at the same viewing distance.

to the right of the platform. The bar was moved slowly (about $5 \mathrm{~mm} / \mathrm{sec}$ ) toward or away from the subject, starting from the far or near end of the parallel in alternation. The bar was always moved while abutting onto the parallel, or, if the parallel was not present, along a corresponding line marked lightly in pencil on the surface of the platform. When the bar was moving it was always upright, with the needles against the cork surface. The subject indicated when the moving bar appeared to be coplanar with the fixed bar on the left. At this point, the bar was pushed down into the cork so that it was held in place by the needles. The subject then indicated whether or not it still appeared to be coplanar, and if not, it was raised and moved again in the direction indicated by the subject until the subject was satisfied with its alignment. Final judgments of alignment were always made when the bar was stationary, with the experimenter's hand well removed from it. The subjects were encouraged to be as certain as possible about these final positions. Misalignment between true and apparent alignment was measured to the nearest millimeter along the right parallel (or the corresponding pencil line). The true alignment was penciled lightly on the cork surface. The subjects were asked to close their eyes while these 
measurements were being made, and the body of the experimenter was always interposed between subject and stimulus object. There were four trials, two beginning from a position at the far end of the parallel and two from the near end, in alternation.

\section{Scoring}

The misalignment score was always the mean of the four differences between true and apparent alignment in millimeters along the axis of the right sagittal bar. Differences in the direction to compensate for the conventional Poggendorff effect when the stimulus object was viewed from directly above were treated as positive, and those in the opposite direction as negative.

\section{EXPERIMENT 1}

The purpose of Experiment 1 was to establish whether, as suggested by earlier informal observations, coplanar bars arranged obliquely at $45^{\circ}$ in depth and intersecting sagittal parallel bars as shown in Figure 1A would appear to be misaligned. Although interest centered mainly on the occurrence and size of the misalignment effect in depth, there was interest also in any similarities between these effects and those in conventional 2-D figures. Therefore, a condition without parallel bars, shown in Figure 1B, was included for comparison. In the 2-D situation, a slight but significant misalignment effect occurs with two $45^{\circ}$ oblique lines presented without the parallels between them, the "paralleless" Poggendorff illusion (Day, 1973; Goldstein \& Weintraub, 1972).

\section{Method}

The dimensions of the oblique and parallel bars are described above. In the stimulus object consisting of both parallel and oblique bars the former were separated by $50 \mathrm{~mm}$ and the latter intersected them at $45^{\circ}$. If extended, the collinear oblique bars would have passed through the center of the space between the parallels. The oblique bars without parallels coincided with those with parallels and were positioned and measured with reference to pencil lines on the cork surface.

\section{Results and Discussion}

Of the 12 mean scores for each of the two stimulus conditions, 11 were positive and 1 negative, with the negative scores deriving from different subjects. The 11 positive scores for the condition with parallel bars ranged from 11.0 to $91.8 \mathrm{~mm}$, and those for the condition without them ranged from 0.5 to $17.5 \mathrm{~mm}$. The negative scores for these two conditions were respectively -20.3 and $-18.0 \mathrm{~mm}$. The mean scores and their standard deviations are shown in Table 1 . It can be seen that the mean for the object with parallels was about seven to eight times greater than that without them. Two $t$ tests showed that whereas the mean for the stimulus object with parallel bars was significant $[t(11)=3.78, p<.01]$, that for the object without them was not $[t(11)=1.76, p>.05]$. A third $t$ test for matched samples showed that the difference between the two means was also significant $[t(11)=$ $3.48, p<.01]$.

The outcomes of this experiment confirmed the earlier, informal observations in showing that there is a relatively large misalignment effect with bars arranged obliquely in depth intersecting sagittal parallel bars at their inner ends. This effect did not occur reliably without the parallel bars. The latter outcome was due to the relatively large negative score of 1 subject.

\section{EXPERIMENT 2}

Accuracy of alignment of oblique bars in depth can be expected to depend on the availability and effectiveness of cues for depth. Intuitively it can be supposed that the more available and effective the cues for depth, the smaller the misalignment effect will be. In other words, on first consideration it seems likely that whatever factors in the stimulus array give rise to apparent misalignment of coplanar elements, those factors would have less influence in the face of numerous and strong cues for depth. In this regard, it could be expected that binocular viewing involving the strong retinal disparity cue would result in a smaller misalignment effect than would monocular viewing without it. Experiment 2 was therefore concerned with apparent misalignment of the coplanar bars in Figure 1A with binocular and monocular viewing.

In consideration of the depth cues available in the arrangement shown in Figure 1, it can be noted that the surface of the platform was not visible from the subject's viewing position, and that the surface of the bars was matte white and virtually textureless. Therefore, texture gradients on the platform and the bars themselves could not have contributed to depth discrimination. With binocular viewing, both retinal disparity and oculomotor cues together with the linear perspectives of the oblique bar outlines could be expected to have been operative. With monocular viewing, retinal disparity was eliminated so that only oculomotor and perspective cues were available.

\section{Method}

Half the subjects viewed the stimuli with one eye first and with two eyes second; the other half viewed them in the opposite order. Head position was also the same as in Experiment 1. Using both eyes, the subjects adjusted their head position on the chinrest so that they were looking down the central axis between the parallel bars. This position was maintained for both the binocular and the monocular viewing conditions. All other aspects of the procedure, including the number of trials, the starting positions, and scoring were the same as before.

\section{Results and Discussion}

For binocular viewing, all 12 misalignment scores were positive; for monocular viewing, 10 of the 12 were positive. For the binocular condition, the scores ranged from 6.5 to $47.8 \mathrm{~mm}$ and for the monocular condition from 9.0 to $15.25 \mathrm{~mm}$. The mean scores and their standard deviations are shown in Table 1, from which it can be noted that the mean for the binocular condition was about five times greater than that for the binocular condition. Separate $t$ tests showed that whereas the mean score for the binocular condition was significant $[t(11)=1.997, p<$ $.05]$, that for the monocular condition was not $[t(11)=$ $1.97, p>.05]$. The difference between the two means was also significant $[t(11)=10.85, p<.001]$. 
Table 1

Mean Misalignments in Milimeters and Standard Deviations for the Six Experiments

\begin{tabular}{|c|c|c|c|}
\hline Experiment & $\begin{array}{c}\text { Stimulus } \\
\text { Conditions }\end{array}$ & $\begin{array}{c}\text { Mean } \\
\text { Misalignment (mm) }\end{array}$ & $S D$ \\
\hline 1 & $\begin{array}{l}\text { with parallel bars } \\
\text { without parallel bars }\end{array}$ & $\begin{array}{r}33.6 \\
4.1\end{array}$ & $\begin{array}{r}29.5 \\
8.3\end{array}$ \\
\hline 2 & $\begin{array}{l}\text { binocular viewing } \\
\text { monocular viewing }\end{array}$ & $\begin{array}{r}26.7 \\
5.7\end{array}$ & $\begin{array}{r}10.8 \\
6.5\end{array}$ \\
\hline 3 & $\begin{array}{l}30^{\circ} \text { oblique angle } \\
45^{\circ} \text { oblique angle } \\
60^{\circ} \text { oblique angle }\end{array}$ & $\begin{array}{l}42.0 \\
31.6 \\
28.2\end{array}$ & $\begin{array}{l}32.2 \\
25.3 \\
26.3\end{array}$ \\
\hline 4 & $\begin{array}{l}50 \mathrm{~mm} \text { between parallels } \\
100 \mathrm{~mm} \text { between parallels }\end{array}$ & $\begin{array}{l}17.4 \\
35.9\end{array}$ & $\begin{array}{l}12.0 \\
21.3\end{array}$ \\
\hline 5 & $\begin{array}{l}45^{\circ} \text {, plain bars } \\
45^{\circ} \text {, patterned bars } \\
90^{\circ} \text {, plain bars } \\
90^{\circ} \text {, patterned bars }\end{array}$ & $\begin{array}{r}12.7 \\
12.1 \\
-2.8 \\
-5.3\end{array}$ & $\begin{array}{r}12.0 \\
11.6 \\
11.6 \\
9.0\end{array}$ \\
\hline 6 & $\begin{array}{l}\text { with parallels } \\
\text { without parallels }\end{array}$ & $\begin{array}{l}8.2 \\
2.5\end{array}$ & $\begin{array}{l}4.0 \\
1.9 \\
\end{array}$ \\
\hline
\end{tabular}

Note-Experiment 6 consisted of 2-D viewing from above.

These outcomes are quite clear in showing that the strong retinal disparity cue for depth does not lead to a smaller misalignment effert. In fact, with binocular viewing, the misalignment effuct was very much greater than it was with monocular viewing. Furthermore, with monocular viewing, the effect was not statistically reliable. Thus the data do not sustain the intuitive expectation based on the availability of cues for depth.

What, then, is the explanation of the strong misalignment effect with binocular viewing and the absence of an effect with monocular viewing? We believe that the answer to this question lies in the marked loss of depth and the altered aspect of the stimulus object with monocular viewing. Postexperimental inspection of the object showed that with binocular viewing the parallel bars appeared to be extended in depth, with both inside surfaces clearly visible and symmetrically convergent. In monocular viewing, the parallel bars were markedly shortened, with only one inside surface visible and only the vertical near end of the other visible. As will be noted below (see General Discussion), apparent misalignment of the oblique bars is attributed to their positions relative to the depthextended parallel bars. Therefore, it can be expected that loss of depth with monocular viewing, a necessary condition for apparent misalignment in depth, is eliminated.

\section{EXPERIMENT 3}

Apparent misalignment of collinear oblique lines in the conventional Poggendorff figure increases as the acute angle to the parallel decreases - that is, as the obliques are rotated toward the axis of the parallels (Anton, 1976; Hotopf, Ollerearnshaw, \& Brown, 1974; Velinsky, 1925; Weintraub \& Krantz, 1971). This inverse relationship generalizes to both acute- and obtuse-angle forms of the figure, and to one consisting of a vertical and an oblique line with a gap between them (MacKay \& Newbigging, 1977). It was therefore of interest to establish whether this relationship would hold for the 3-D stimulus object as the angles in depth between the oblique and parallel bars were varied. Experiment 3 was concerned with this issue.

\section{Method}

The linear dimensions of the oblique and parallel bars were the same as those described above. The acute angles between the oblique and the parallel bars were $30^{\circ}, 45^{\circ}$, and $60^{\circ}$ and the obtuse angles, accordingly, were $150^{\circ}, 135^{\circ}$, and $120^{\circ}$. Each subject was presented with these conditions in random order. All four adjustments to apparent alignment for one condition were completed before the next was presented.

\section{Results and Discussion}

All 12 scores for the $30^{\circ}$ condition, 11 of the 12 for the $45^{\circ}$ condition, and 10 of the 12 for the $60^{\circ}$ condition were positive and the others negative. The ranges of scores for these three conditions were, respectively, +3.0 to $+85.3 \mathrm{~mm},-10.0$ to $+71.8 \mathrm{~mm}$, and -17.3 to $+69.5 \mathrm{~mm}$. The mean misalignment effects and their standard deviations for the three conditions are shown in Table 1 . It can be noted that the mean for the $45^{\circ}$ acute angle was similar to that for the same angle in Experiment 1 . It can also be noted that the effects for $30^{\circ}$ and $60^{\circ}$ were respectively greater and smaller. All three means were significantly different from zero $\left[t_{30}(11)=4.52\right.$, $p<.001 ; t_{45}(11)=4.32, p<.01 ; t_{60}(11)=3.71, p<$ $.01]$. A one-way analysis of variance (ANOVA) showed that the three means were not significantly different from one another $[F(2,22)=0.99, p>.05]$.

Thus, although all three angles resulted in significant misalignment effects, and although the trend in their size was in the expected direction for the 2-D figure, the differences between them were not statistically different. However, before concluding that the size of the misalignment effect does not vary with the oblique-parallel angle, consideration must be given to the possibility that the angles themselves might not have been clearly discriminable and that the relatively high variance might have masked any effect due to angle. These possibilities are considered respectively in the introduction to Experiment 5 and in the General Discussion.

\section{EXPERIMENT 4}

Apparent misalignment in the conventional Poggendorff figure increases as a linear function of the separation between the parallels (Burmester, 1896; Day \& Dickinson, 1976; Hotopf \& Ollerearnshaw, 1972; Weintraub \& Krantz, 1971). It was of interest, therefore, to establish whether the apparent misalignment of oblique coplanar bars would also increase with separation between the parallel bars. Experiment 4 was designed for this purpose, with separations of $50 \mathrm{~mm}$ (as in Experiments 1, 2, and 3) and $100 \mathrm{~mm}$. 


\section{Method}

The stimulus object was the same as that in Experiment 1, with the exception that under one condition the parallel bars were $50 \mathrm{~mm}$ apart, and under the other, $100 \mathrm{~mm}$. The order of presentation of these conditions alternated over the 12 subjects.

\section{Results and Discussion}

All 12 scores in the two conditions were positive. The range for those in the 50-mm condition was $0.3-37.5 \mathrm{~mm}$, and that for the $100-\mathrm{mm}$ condition, $0.5-79.8 \mathrm{~mm}$. The means and standard deviations are shown in Table 1 . The mean for the $100-\mathrm{mm}$ condition was about double the size of that for the $50-\mathrm{mm}$ condition. Surprisingly, the mean for the latter was only about half that for the same condition in Experiments 1 and 2. Two $t$ tests showed that both means were significantly greater than zero $\left[t_{50}(11)=\right.$ $\left.5.03, p=.001 ; t_{100}(11)=5.83, p<.001\right]$, and a third for matched samples showed that they were different from each other $[t(11)=2.83, p<.02]$.

The reduction in the mean size $(17.4 \mathrm{~mm})$ of the misalignment effect for the 50 -mm-separation condition in this experiment to markedly less than those $(33.6,26.7$, and $31.6 \mathrm{~mm}$ ) for the same condition in Experiments 1, 2 , and 3 was unexpected. Thus, although the mean scores were significantly different and in the direction expected from the effect of separation between the parallels for the 2-D effect, the reduction for the 50-mm condition compared with the means for the same condition in Experiments 1,2 , and 3 calls for some caution in interpreting this result. This issue is considered below in the General Discussion.

\section{EXPERIMENT 5}

The bars intersecting the parallels in the preceding experiments were always oriented obliquely in depth. The condition in which the two bars were at right angles to the parallels was not previously included. In the conventional Poggendorff figure, no misalignment occurs when the transversal lines are at right angles to the parallels (Velinsky, 1925; Weintraub, Krantz, \& Olsen, 1980). It was therefore of interest to establish whether this would also be the case with the 3-D object viewed in depth.

It is conceivable that the high variance both within and between the earlier experiments was due to difficulty in discriminating the angles between the oblique and parallel bars. Therefore, in Experiment 5 a regular pattern was added to the near surfaces of the oblique bars in order to provide a texture-gradient cue for their oblique orientation in depth and so reduce possible fluctuations in the apparent size of the oblique-parallel angles. The size and variation of misalignment effects with and without this cue were compared. Thus there were four conditions, bars with and without a surface pattern intersecting the parallels at $45^{\circ}$ and $90^{\circ}$.

\section{Method}

The near surfaces of one pair of bars to be aligned were uniformly white as before; those of the other pair were patterned with a promi- nent grid of intersecting dark blue lines. Strips of adhesive "Contact" "with the grid pattern were stuck to the bars to form a regular and symmetrical pattern of 12.5 -mm squares along the lengths of the bars. The lines forming the pattern were $1.5 \mathrm{~mm}$ thick and were clearly visible from the viewing position. The four conditions involving plain and patterned bars at angles of $45^{\circ}$ and $90^{\circ}$ were presented in a different random order to each subject.

\section{Results and Discussion}

Of the 12 scores for each condition, the number that were positive for the two angles $\left(45^{\circ}\right.$ and $\left.90^{\circ}\right)$ with plain (pl) and patterned (pa) bars were $45^{\circ}$-pl, $10 ; 45^{\circ}$-pa, 10; $90^{\circ}$-pl, 6; and $90^{\circ}$-pa, 3. The ranges of scores for these four conditions were, respectively, $-4.8-37.0 \mathrm{~mm}$, $-2.3-35.0 \mathrm{~mm},-30.0-11.5 \mathrm{~mm}$, and $-21.0-3.8 \mathrm{~mm}$. The mean misalignment scores and their standard deviations are shown in Table 1. The means for the plain and patterned bars at $45^{\circ}$ to the parallels were both positive, and those for the same bars at $90^{\circ}$ were both negative. Separate $t$ tests showed that the means for the two $45^{\circ}$ conditions were significant $\left[t_{\mathrm{p} 1}(11)=3.65, p<.01\right.$; $\left.t_{\mathrm{pa}}(11)=3.60, p<.01\right]$ and that those for the two $90^{\circ}$ conditions were not $\left[t_{\mathrm{pl}}(11)=0.85, p>.05 ; t_{\mathrm{pa}}(11)=\right.$ $2.04, p>.05]$. A two-way ANOVA, in which the main factors were the oblique-parallel angle (A) and the surface pattern $(\mathrm{P})$ of the bars, showed that the former was significant $\left[F_{\mathrm{A}}(1,11)=12.47, p<.01\right]$ and that the latter was not $\left[F_{\mathrm{P}}(1,11)=0.47, p>.05\right]$. The interaction between these factors also failed to reach significance $[F(1,11)=0.32, p>.05]$. It can be seen from Table 1 that the standard deviations were about the same for the plain and the patterned bars.

In summary, the addition of a regular pattern to the near surfaces of the bars did not influence the misalignment effect, which was again evident when the bars were at $45^{\circ}$ to the parallels but absent when they were at $90^{\circ}$. The absence of any effect due to pattern is not in accord with the results reported by Daniels and Gordon (1993). However, it can be noted that both the stimulus conditions and the fineness of the surface texture were different for the two experiments. It can also be noted that the misalignment effect for the $45^{\circ}$ plain oblique bars $(12.7 \mathrm{~mm})$ was smaller than that for the same condition in Experiment 4 $(17.4 \mathrm{~mm})$ and considerably less than those for the same conditions in Experiments 1, 2, and 3 (33.6, 26.7, and $31.6 \mathrm{~mm}$, respectively). These wide fluctuations in apparent misalignment and their implications for the reliability of the differences between experimental conditions are discussed below.

\section{EXPERIMENT 6}

Viewed from above, the upper edges of the 3-D stimulus object in Figure 1A form a conventional Poggendorff figure, and the oblique transversal edges appear to be misaligned in the usual way. It is clearly of interest to compare the apparent misalignment in depth found in the five preceding experiments with that found for the same object at the same distance, viewed from above. Therefore, 
in Experiment 6, subjects were required to indicate when the two $45^{\circ}$ oblique bars appeared to be aligned while looking down on the objects shown in Figures $1 \mathrm{~A}$ and $1 \mathrm{~B}$. The viewing distance of $100 \mathrm{~cm}$ was the same as that for the in-depth arrangement in the earlier experiments.

\section{Method}

The subjects stood on a set of library steps in front of the platform and looked down on the stimulus objects. A meter ruler was used to check that the subjects eyes were as near as possible to $100 \mathrm{~cm}$ above the center of the objects. The stimulus objects were the same as before, with the bars at $45^{\circ}$ to the parallels. The procedure for adjusting the bar on the right so that it appeared to the subject to be aligned with that on the left was the same as that in the earlier experiments.

\section{Results and Discussion}

The mean misalignments for the two objects are shown in Table 1. It can be seen that the mean for the object with parallels $(P)$ was about three times greater than that for the object without parallels (NP). Both means were significant $\left[t_{\mathrm{P}}(11)=7.00, p<.001 ; t_{\mathrm{NP}}(11)=4.56\right.$, $p<.001]$. The difference between these means was also significant $[t(11)=6.12, p<.001]$. In an earlier experiment (Day, 1973), the mean misalignments for Poggendorff figures consisting of parallel lines with $45^{\circ} \mathrm{ob}$ liques and of the obliques alone were, respectively, 7.26 and $2.17 \mathrm{~mm}$, values that more or less accord with those for the stimulus objects in this experiment. It is clear from these results that the mean misalignment effect for the 2-D Poggendorff figure formed by the upper edges of the 3-D stimulus object is much smaller than that in depth, and the variance is less. Furthermore, the effect is evident when the parallels are removed. In brief, a smaller and more stable misalignment effect than that in depth occurs when the edges of the 3-D object are viewed from above.

\section{GENERAL DISCUSSION}

The data from Experiments 1-5 show that coplanar bars arranged obliquely in depth with each intersecting one of two parallel sagittal bars appear misaligned in depth when viewed with both eyes. This effect was robust over five experiments. Taking into account all conditions involving the complete stimulus object in binocular view, the frequencies of positive individual scores for Experiments 1, $2,3,4$, and 5 were, respectively, $41 / 48,128 / 144,84 / 96$, $77 / 96$, and $46 / 48$.

The mean scores in Table 1 show that the effect was eliminated when the oblique bars were presented without the parallel bars (Experiment 1) and when the complete object was viewed monocularly (Experiment 2); that the effect did not vary significantly over oblique-parallel angles of $30^{\circ}, 45^{\circ}$, and $60^{\circ}$ (Experiment 3); that it approximately doubled in size when the separation between the parallel bars was increased from 50 to $100 \mathrm{~mm}$ (Experiment 4); that it was unaffected by the presence of a regular pattern on the visible surfaces of the oblique bars; and that it was absent when the latter were oriented at $90^{\circ}$ to the parallels (Experiment 5).
Some consideration must first be given to the variation in the size of the effect for the same condition over the five experiments. When the oblique-parallel angle was $45^{\circ}$, the separation between the parallels was $50 \mathrm{~mm}$, and viewing was binocular, the mean effects in Experiments 1, $2,3,4$, and 5 were $33.6,26.7,31.6,17.4$, and $12.7 \mathrm{~mm}$, respectively. Thus, in Experiment 5, the size of the effect was almost one third of that in Experiment 1. A possible basis for this inconsistency is suggested by the ratios of means to standard deviations for the two oblique bars alone (Experiment 1) and the two oblique edges alone (Experiment 6)-that is, between the 3-D and 2-D effects. The ratios for Experiments 1 and 6 were 4.1:8.3 and 2.5:1.9, respectively. Leibowitz (1955) has pointed out that in vernier acuity tasks, the lower the variability the greater the acuity for misalignment. That is to say, variability serves as a measure of visual acuity. The task of adjusting two oblique elements to apparent alignment is essentially a vernier acuity task; the elements are adjusted so that no misalignment is visible, rather than, as is usual, so that a misalignment is just visible. In these terms, the greater variance for the alignment of two bars in depth than that for two edges in the same plane indicates much poorer acuity for the former. Thus it can be concluded that vernier acuity for bars in depth is markedly poorer than that for lines on a surface. It is reasonable to assume that the wide fluctuations in mean misalignment effects were due to sampling differences. There are considerable individual differences in ability to align elements obliquely in depth, and these differences are reflected in the differences between the sample means.

As pointed out in the introduction, these experiments originated with an explanation of the conventional form of the Poggendorff illusion in terms of a perceptual compromise. Although the oblique lines are positioned in an oblique axis, they are separated from each other along the vertical axes of the parallels. The slight but persistent misalignment effect is held to reflect a compromise in perception between these two axis-relative positions.

We take the view that the apparent misalignment of oblique coplanar bars in depth reported here can be explained in the same terms. Although the bars shared the same oblique plane, they were displaced from each other in depth along the sagittal axes of the parallel bars. In consequence, the oblique bars appear to be slightly displaced from each other with the bar on the right appearing slightly too far for oblique alignment with that on the left. In other words, we suggest that the states of alignment of two elements in one axis and their displacement from each other in another axis gives rise to a perceptual compromise between these two states of affairs in both 2-D and 3-D space.

\section{REFERENCES}

ANTon, B. S. (1976). Poggendorff illusion as a function of orientation of transversal and parallel lines. Perceptual \& Motor Skills, 43, 83-90. Burmester, E. (1896). Beiträge zur experimentellen Bestimmung geometrischoptischer Täuschungen. Zeitschrift Für Psychologie, 18, 355-394. 
Daniels, V., \& Gordon, I. E. (1993). Occlusion and the distortion of alignment in 3D space. Perception, 22, 1005-.

DAY, R. H. (1973). The oblique line illusion: The Poggendorff effect without parallels. Quarterly Journal of Experimental Psychology, 25, 535-541.

Day, R. H., \& Dickinson, R. G. (1976). The components of the Poggendorff illusion. British Journal of Psychology, 67, 537-552.

DAY, R. H., \& PARKer, A. L. (in press). On apparent misalignment of collinear edges and boundaries. Perception \& Psychophysics.

DAY, R. H., \& PARKs, T. E. (1989). To exorcise a ghost from the perceptual machine. In M. Hershenson (Ed.), The moon illusion (pp. 343350). Hillsdale, NJ: Erlbaum.

Day, R. H., Stecher, E. J., \& Parker, A. L. (1993). The effect of edge orientation and movement direction on the apparent misalignment of collinear bars. Perception \& Psychophysics, 53, 642-647.

Goldstein, M. B., \& Weintraub, D. J. (1972). The parallel-less Poggendorff: Virtual contours put the illusion down but not out. Perception \& Psychophysics, 11, 353-355.

Gordon, I. E., DAY, R. H., \& DoRWARd, F. (1993). A Müller-Lyer illusion for egocentric distance with 3D stimulus objects. Scandinavian Journal of Psychology, 34, 97-106.

Hotopf, W. H. N., Ollerearnshaw, C. (1972). The regression to right angles tendency and the Poggendorff illusion. II. British Journal of Psychology, 63, 369-379.
Hotopf, W. H. N., Ollerearnshaw, C., \& Brown, S. (1974). The regression to right angles tendency and the Poggendorff illusion. III. British Journal of Psychology, 65, 213-231.

LeiBowitz, H. (1955). Some factors influencing the variability of vernier adjustments. American Journal of Psychology, 68, 266-272.

Mackay, D. C., \& Newbigging, P. L. (1977). The Poggendorff and its variants do arouse the same perceptual process. Perception \& Psychophysics, 21, 26-32.

Velinsky, P.S. (1925). Explication physiologique de l'illusion de Poggendorff. Année Psychologique, 26, 107-116.

Weintraub, D. J., \& Krantz, D. H. (1971). The Poggendorff illusion: Amputations, rotations, and other perturbations. Perception \& Psychophysics, 10, 257-264.

Weintraub, D. J., Krantz, D. H., \& Olsen, T. P. (1980). The Poggendorff illusion: Consider all the angles. Journal of Experimental Psychology: Human Perception \& Performance, 6, 718-725.

(Manuscript received November 2, 1992; revision accepted for publication January 13, 1994.) 